\title{
POST-MORTEM RADIOGRAPHY AND GASEOUS FIXATION OF THE LUNG
}

BY

\author{
R. J. R. CURETON AND DAVID H. TRAPNELL \\ From the Departments of Pathology and Diagnostic Radiology, St. Bartholomew's Hospital, London
}

(RECEIVED FOR PUBLICATION OCTOBER 4, 1960)

From time to time radiologists see pulmonary shadows in chest radiographs, the exact nature of which is uncertain. When material from such cases becomes available for histological examination there is often no exact correlation made between the shadow seen radiologically and the lesion examined histologically, particularly if the lesions are multiple or if several different abnormalities are present together. The technique we describe has helped us to obtain greater precision in the study of radiological abnormalities by histological methods. We have also used it for the study of small previously unsuspected lesions which have been located by this radiographic method and which would probably have escaped attention if the lung had been sliced at random.

Post-mortem radiography of uninflated lung is unsatisfactory because few radiographic details of anatomical structure or of pathological change can be demonstrated (Fig. 1a), and, furthermore, the histological details in uninflated lung tissue are usually difficult to interpret and may be frankly misleading. Such radiographs are particularly unsatisfactory if the lesions as well as being small have low radiographic density.

Various radiographic methods of investigating cadaveric lungs have been used in the past, but they all have had severe limitations. Allred and Garland (1935), Beilin, Fink, and Leslie (1951), and Greening and Pendergrass (1954) have described radiography of the chest of the cadaver suspended in an upright position, but such methods are inconvenient and provide little additional information. Cunningham and Miller (1952) described a technique for the radiography of slices of lungs fixed with formalin solution, using special low-voltage $x$-ray apparatus. Apart from the need for special equipment, this method does not allow any real comparison of the ante-mortem and postmortem radiographs. Cicero and Celis (1955) attempted to demonstrate the vascular changes occurring in pulmonary tuberculosis by a technique in which the lungs were inflated with air and radiographs were made after a barium suspension had been injected into the pulmonary artery. However, they apparently made no $\infty$ radiographs of the inflated lung before injection of the contrast medium.

We have traced two descriptions of radiography of lungs inflated with air after removal from the body. Greening and Pendergrass (1954) used this method but did not mention the correlation that the technique makes possible between the shadow seen by the radiologist and the lesion examined by the pathologist. Few details of their method are given and no histological findings are described. Oderr, Pizzolato, and Ziskind (1958) published an account of a technique for drying lungs in the inflated position, slicing the dried lungs, and radiographing the sections. They give a very scanty description of their technique and appear to have made no investigation into the effects of drying the lung. We feel that their method has two fundamental weaknesses: first, the lung tissue is desiccated and thus some of the apparent radiological or histological changes may be artefacts; and, second, special $x$-ray apparatus is necessary to make possible radiography at $10 \mathrm{kV}$, thus making their method of little value to most radiologists or pathologists working in ordinary hospital departments.

Inflation of a lung with air restores it to approximately its former size and shape and makes possible a comparison of the subsequent radiographs with the ante-mortem chest radiographs, thus greatly enhancing the value of postmortem radiography (Fig. 1b and 1c). Radiographs of air-inflated lung reveal in detail the structure of the lung and the presence and extent of pathological changes within it. No unusual apparatus is necessary for the inflation of the lungs, and the subsequent radiography can be carried out with a portable $x$-ray machine or with apparatus available in any radiological department. However, even if radiographs of the inflated lung are taken in two planes it may be hard to locate very small lesions in a lung. As an 

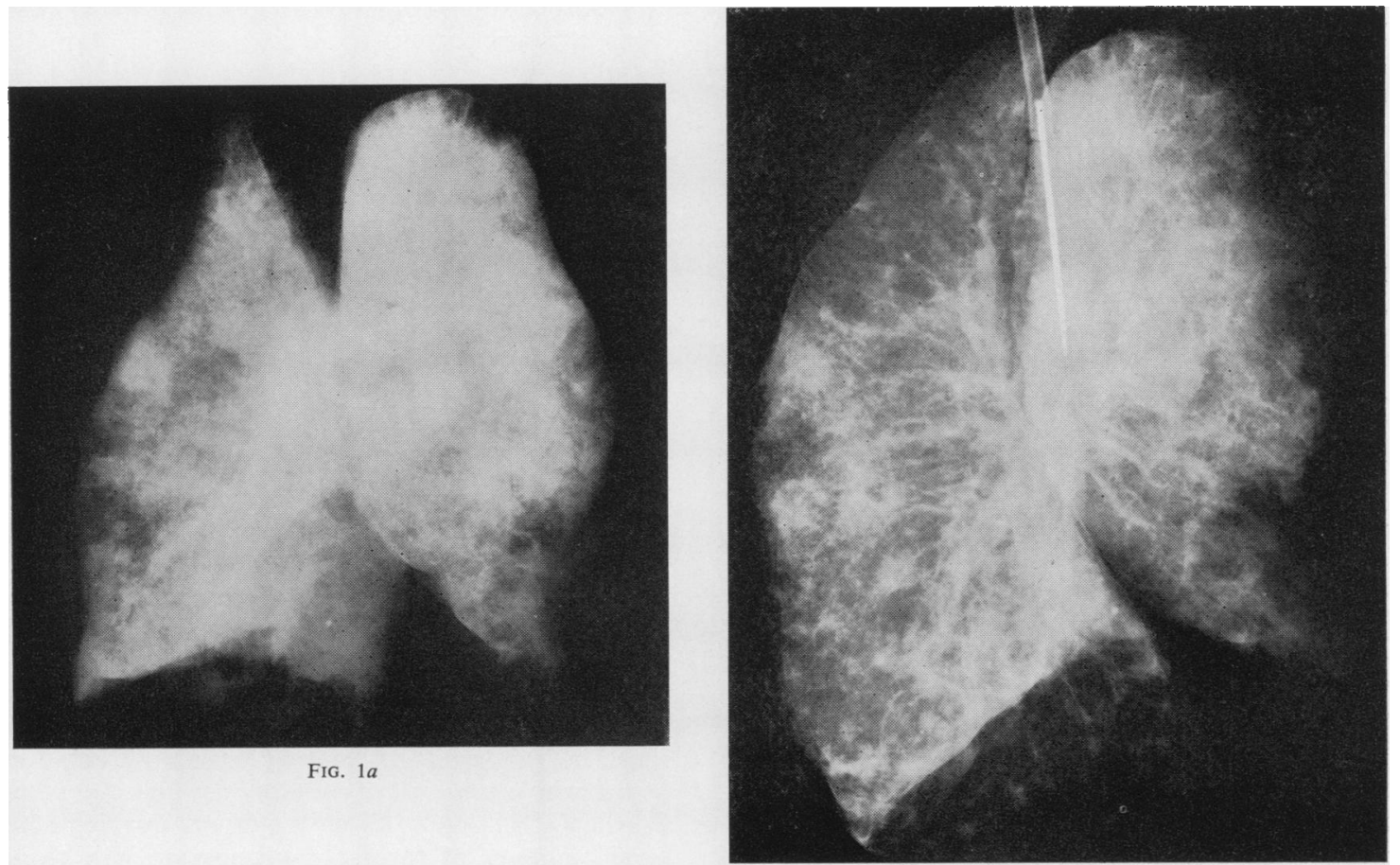

FIG. $1 b$

Fig. 1.-Multiple metastatic deposits from a carcinoma of the breast in a woman aged 62 years. (a) Radiograph of the left lung (uninflated) immediately after removal from the body. (b) Radiograph showing the details of structure revealed by inflation (with formaldehyde gas). ( $c$ ) Radiograph showing part of a slice (approximately $1 \mathrm{~cm}$. thick) of the upper part of the upper lobe showing tumour deposits. One metastasis (ringed) was removed for section. The clarity of the blood vessels is due to the contrast with the adjacent gas-filled alveoli.

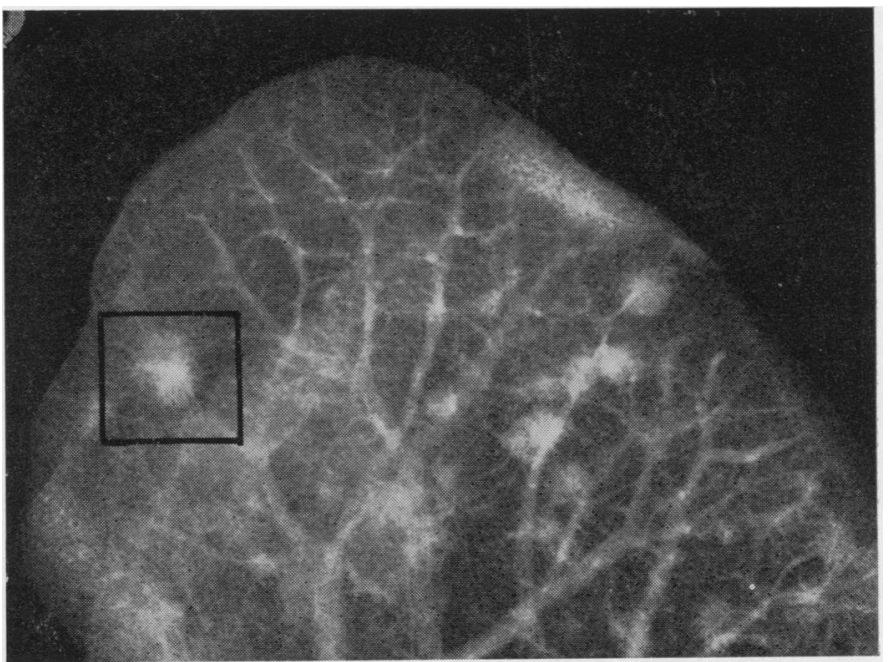

Fig. 1c 


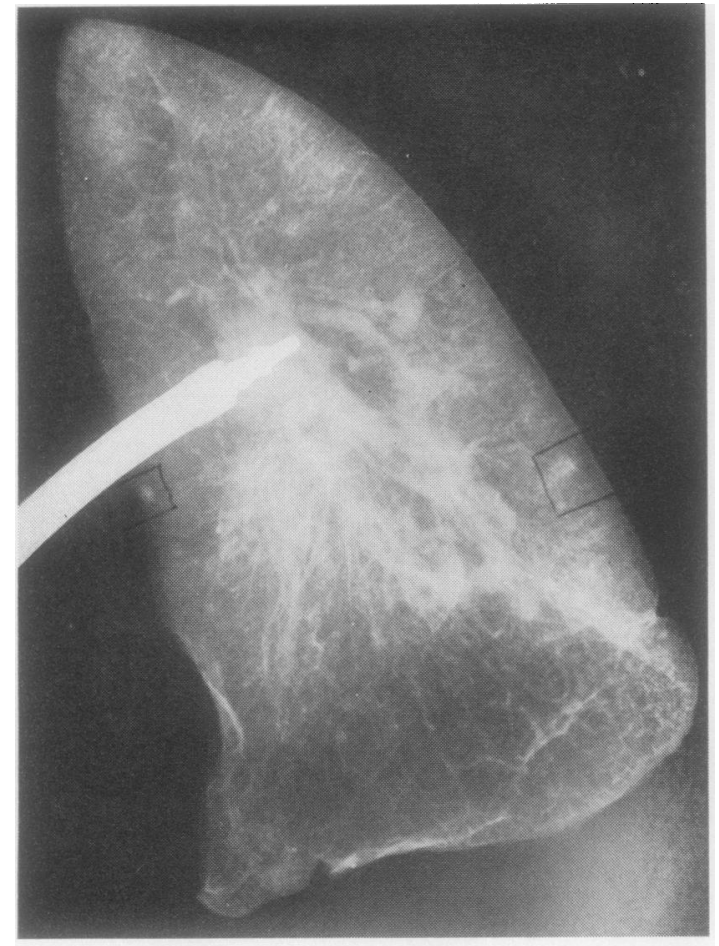

adjunct in such cases it may be necessary to make radiographs of slices of the lung after fixation.

We have found that, if the lung is inflated with air and subsequently fixed by injecting formalin solution into the bronchial tree, further useful radiography of the whole lung is impossible (Fig. 2b). Clearly what is needed is gaseous fixation to permit interpretation of the radiographs of the whole lung (or slices of it) after fixation. It was for this purpose that the method we describe here was devised.

After we had begun this work three reports of fixation of the lung by gas were published in America. All describe the use of a flow of air through formalin solution to fix the lung. Jones (1960) used a mixture of vapour from $50 \%$ ethyl alcohol and $50 \%$ formalin, but concluded that this method was impracticable because of the time involved and the unreliability of the technique.

Blumenthal and Boren (1959) state that, to maintain the lung in a state of complete inflation, a flow of 5 to 10 litres per minute was necessary, and that, as there was a constant leak from the lung surface, inflation pressures could not be measured. In our experience, the lung can and must be rendered air-tight because full inflation of the whole lung is not usually possible otherwise. Furthermore, such large air-flows through the small volumes of formalin solution used by these authors would, we feel, have produced at the end of three to five days an inflation mixture containing very little formaldehyde vapour. Pratt (1959) describes a very similar method, but inflated the lung with formaldehyde vapour for only 18 hours. None of these papers describes the value of radiography of the lung after inflation.

Each of these methods involved desiccation of the lung by blowing air through it for about a week after fixation. Apparently only Blumenthal and Boren (1959) have tried to assess the effects of this drying of the lung, and they state that cellular detail in stained sections is excellent, but we find it hard to believe that lung tissue fixed and dried over a period of 10 to 12 days should show no artefacts. Unfortunately it is impossible to assess the quality of fixation from the photomicrographs reproduced in their article.

We realize that such methods may well be of value for demonstrating the coarse changes present in a lung, but if detailed histological investigations are required we feel that a method which does not include desiccation would be an advantage.

FIG. 2.-(a) Radiograph of a left lower lobe (only) inflated with air, showing two small opacities. (b) Radiograph of the same lobe after intrabronchial injection of formaldehyde solution. Further useful radiography is impossible. 


\section{METHOD}

The lung is carefully removed from the body and separated from the mediastinal structures, the main bronchus being divided at the carina. If pleural adhesions are very dense some of the parietal pleura may have to be removed together with the lung. It is most important to preserve the pleura intact if possible, because even very small holes in it can cause troublesome leaks when the lung is inflated. If holes are present in the pleura it may be possible to tie them off in the same way that bleeding points are ligated during an operation. The lung is immersed in water to locate small leaks until it has been rendered airtight. In some cases where the pleura has been severely damaged, we have found that inflation of only one lobe may still be of value if it can be separated from the other lobes (Fig. 2a).

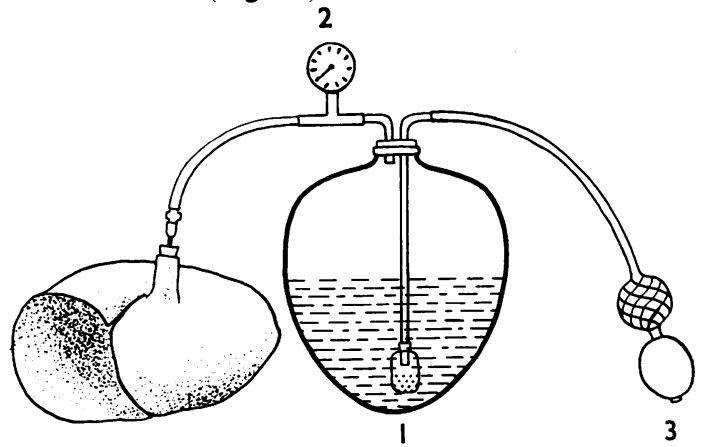

FIG. 3.-Diagram of the apparatus used for inflating lungs with formaldehyde gas. 1, Carboy of concentrated formaldehyde solution; 2, aneroid type of pressure gauge; 3 , rubber syringe.

Formaldehyde gas is used for inflation and fixation. This is obtained by using the vapour above the fluid in a half-empty carboy of concentrated (40\%) formaldehyde solution. The half-empty carboy was used to provide a large surface area of solution and a large volume of gas. A device to make the replacing air form a stream of fine bubbles has been used (a small polythene bottle perforated with numerous small holes) to minimize dilution of the gas in the carboy (Fig. 3).

We have tried several methods of inflation, and consider the best to be by means of a serum needle through a rubber " cork," with which the bronchus is occluded. The rubber "cork," with the needle through it, is tied in position by a ligature round the bronchus and the lung gently inflated by means of a convenient syringe. Inflation pressures should be kept to a minimum to avoid damage to the lung: we have found pressures of $40 \mathrm{~mm}$. mercury or less, up to a maximum of $50 \mathrm{~mm}$., to be satisfactory. Pressures of $50 \mathrm{~mm}$. to $80 \mathrm{~mm}$. $\mathrm{Hg}$ were tried in a few cases without damage except in one instance when the pleura spontaneously stripped from part of the mediastinal surface of the lung at a pressure of just under $60 \mathrm{~mm}$. The needle can be conveniently sealed after inflation of the lung by the "male" component of a tubing connexion, the lumen of which has been closed with solder.

If filling was slower in one part of the lung than the remainder, that part was gently massaged to displace gas back up to the bronchi supplying the area and the lung then reinflated. When inflation was easy and rapid, one filling with gas was sufficient to fix the lung. In cases of pneumonia or oedema we have used several fillings of the lung, allowing it to empty in between each by its own elasticity, to ensure as high a concentration of the gas within the lung as possible.

In two of our cases inflation proved extremely difficult, almost certainly due to the tenacious mucus that was present in the bronchi. Small central areas of the parts poorly inflated were incompletely fixed in both instances.

It is important to ensure a high concentration of formaldehyde in the solution. At one stage, after a year's continuous use of one carboy, four consecutive specimens had small central areas which were incompletely fixed and the strength of the formalin was found to have fallen to $18 \%$. The effect of this deterioration was probably increased by a low room temperature at the time with consequent poor vaporization. Presumably $20-25 \%$ is the minimum strength that can be effectively used.

After inflation, radiographs are taken. Those illustrated here were all taken on Ilfex non-screen film with a Watson MX2 machine with a $1.5 \mathrm{~mm}$. focal spot. The tube has been operated at $54 \mathrm{kV}$, approximately $15 \mathrm{~mA}$, and an anode-film distance of 40 inches. The whole lung is then floated on diluted formalin solution (approximately $20 \%$ ) and covered with a cloth soaked in the fluid. Fixation appears to be rapid and certainly is complete after two to three days. (We have not cut our specimens less than 48 hours after inflation.) If necessary the lung can be left in the formalin for longer periods (in one instance no complication followed leaving the lung thus for five weeks).

If necessary, after fixation the lung can be radiographed again whole or cut into slices (Fig. 1c). By direct comparison of the specimen with the radiographs it is possible to find any particular part of the lung without difficulty unless the lesion sought is extremely small. The area removed for histological examination can be recorded permanently by writing on the radiograph. Very small abnormalities that would almost certainly escape attention by normal random slicing can be located by this method (Fig. $4 a, b$, and $c)$. We believe that the little extra work involved is fully justified by the results obtained and that this method of investigation should be employed whenever detailed examination of a lung is indicated.

\section{RESULTS}

Fixation by this method has proved to be superior to any other we have met so far. Of the 42 cases in which we have used this method seven have been incompletely fixed, the fixation in the 


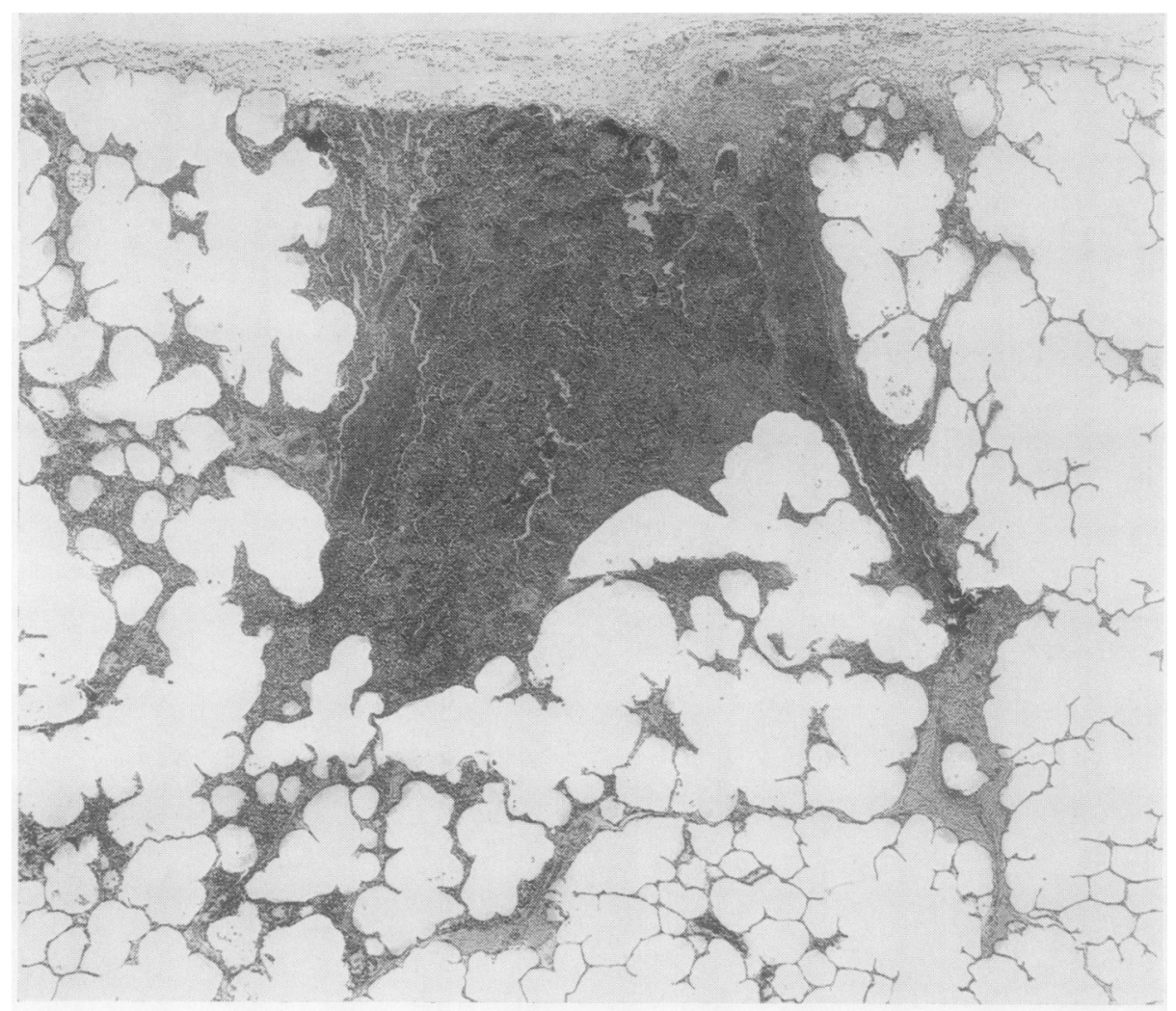

FIG. $4 b$

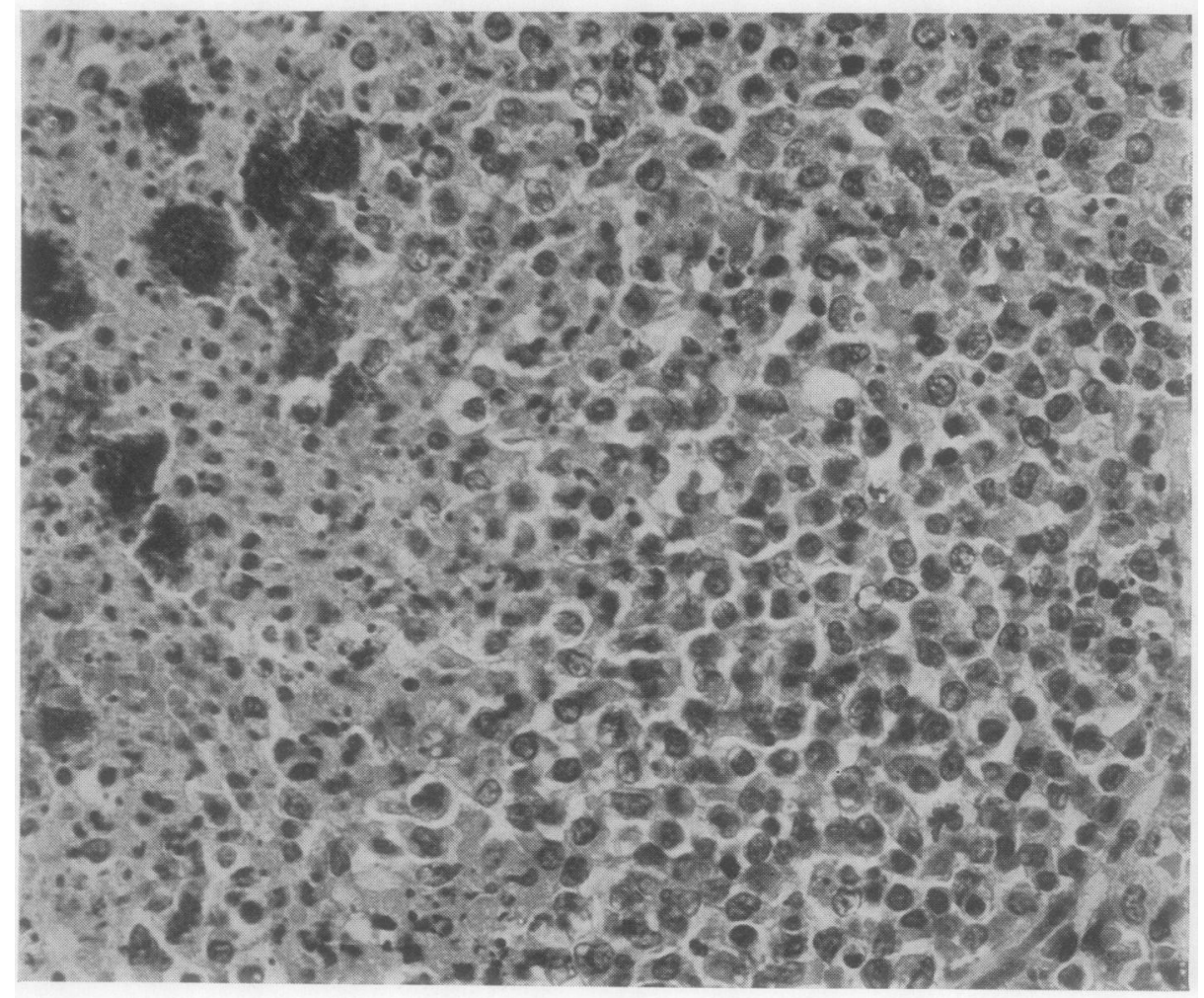

FIG. $4 c$

FIG. 4.-Acute myeloblastic leukaemia in a boy of 13 years. (a) Part of a radiograph of a whole lung showing a small opacity. This lesion was removed for histological section. (b) Low-power photomicrograph ( $\times 28.5$, haematoxylin and eosin) showing subpleural leukaemic infiltration. (c) High power view ( $\times 450)$ of the central part of the lesion. The dark areas in the left of the field are clumps of bacteria. 


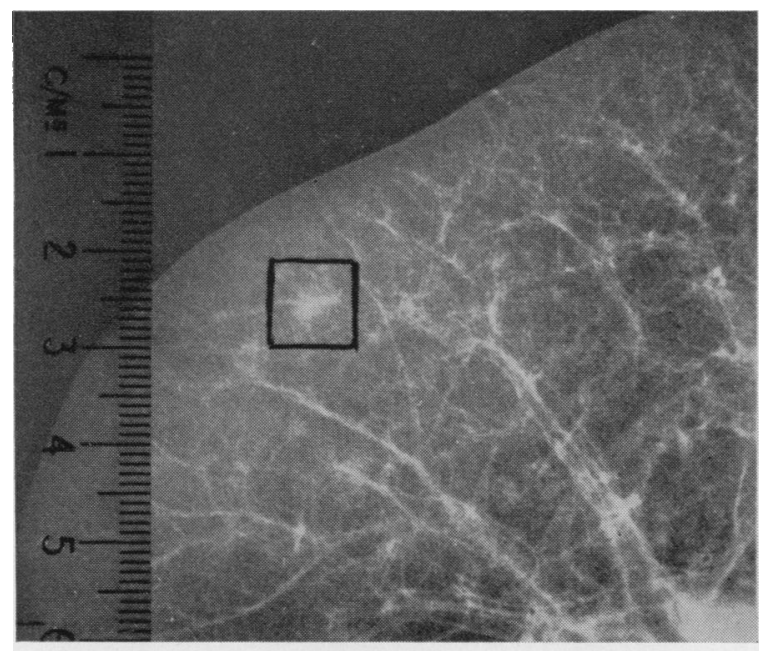

FIG. $4 a$

remainder being excellent. Of these seven cases, the two with thick mucus in the bronchi and the four inflated with a low concentration of formalin gas have already been mentioned. The other was a lung in which there was a large solid haemorrhagic infarct, the central part of which was not completely fixed. Two further lungs, which had several irreparable leaks in them and needed several inflations and had been inflated from the same carboy on the same occasion, had small central areas of imperfect fixation. We think that this was probably due to the dilution of the gas in the carboy by a larger quantity of air than normal. Since then only one lung has been inflated on any one day and the trouble has not recurred. With these exceptions the method has proved successful in a wide range of conditions including bronchopneumonia with pus in the bronchi, confluent pneumonic consolidation, emphysema, Hodgkin's disease with enlarged hilar lymph nodes, and carcinomatosis. In one instance the efficiency of the method was tested on a lung that had already undergone a considerable amount of autolysis. Fixation and arrest of the decomposition proved to be complete.

The fact that fixation was less than perfect in a few cases did not in any way detract from the value of the inflation and subsequent radiography and histological examination, the unfixed areas being small. We consider that careful inflation and elimination of leaks, using a carboy of strong formaldehyde solution, results in fixation quite as good as, or better than, that obtained by other conventional methods of fixation.

\section{SUMMARY}

A simple method for fixing lungs with formaldehyde gas is described which produces fixation often superior to that of other conventional methods. It permits radiography of the lung before and after fixation, which makes possible the detection and localization of small lesions that might be missed on random slicing of the lung, and it enables a correlation to be made between the radiographic and histological appearances.

We are indebted to Mr. J. W. Miller for the preparation of the histological sections, to Mr. P. J. Crocker for the photomicrographs, and to the Department of Medical Photography, St. Bartholomew's Hospital, for the reproductions of the radiographs.

\section{REFERENCES}

Allred, W. L., and Garland, L. H. (1935). Amer. J. Roentgeriol., 33, 839.

Beilin, D. S., Fink, J. P., and Leslie, L. W. (1951). Radiology, 57, 361. Blumenthal, R. J., and Boren, H. G. (1959). Amer. Rev. Tuberc., 79, 764

Cicero, R., and Celis, A. (1955). Ibid., 71, 810.

Cunningham, G. J., and Miller, J. W. (1952). Thorax, 7, 170.

Greening, R. R., and Pendergrass, E. P. (1954). Radiology, 62, 720.

Jones, E. (1960). Amer. Rev. Resp. Dis., 82, 704.

Oderr, C. P., Pizzolato, P., and Ziskind, J. (1958). Radiology, 71, 236.

Pratt, P. C. (1959). Amer. Rev. Tuberc., T9, 842. 